\title{
ON THE PRODUCT OF DIRECTED GRAPHS
}

\section{H. McANDREW}

1. Introduction. The concept of the product of two graphs [1, p. 23] has application in the theory of games and the theory of automata. Weichsel [2] deduced a necessary and sufficient condition for the product of two connected undirected graphs to be connected; he further proves that the product graph, if not connected, has exactly two components. In this paper we deduce the corresponding results for directed graphs, namely that if $G_{1}, G_{2}, \cdots, G_{r}$ are strongly connected directed graphs then $G_{1} \times G_{2} \times \cdots \times G_{r}$ has exactly

$$
\frac{d\left(G_{1}\right) \cdot d\left(G_{2}\right) \cdots d\left(G_{r}\right)}{\operatorname{lcm}\left(d\left(G_{1}\right), d\left(G_{2}\right), \cdots, d\left(G_{r}\right)\right)}
$$

components where $d(G)$ is as defined below.

2. Definitions. We define a directed graph $G$ to be a set of nodes $N(G)$ and a set of edges $E(G)$, the elements of $E(G)$ being ordered pairs $(p, q)$ with $p, q \in N$.

We write $p \Rightarrow q$ if $(p, q) \in E$.

We define a path $P$ of $G$ as a finite sequence $\left\{p_{i}\right\},(0 \leqq i \leqq n, n \geqq 1)$ such that $p_{i} \Rightarrow p_{i+1},(0 \leqq i \leqq n-1)$. We define $n(\mathcal{P})=n$, the number of edges in $\rho$ and write $\rho(p, q)$ for a path with $p_{0}=p, p_{n}=q$.

By $p \in \mathcal{P}$ we mean $\mathcal{P}$ is the path $\left\{p_{i}\right\}$ and for some $i, p_{i}=p$.

We write $p \rightarrow q$ if either $p=q$ or there is a path $\rho(p, q)$.

We say $p, q$ are strongly connected if $p \rightarrow q$ and $q \rightarrow p$. This is clearly an equivalence relation; we define a component $C$ of $G$ to be a subgraph whose nodes, $N(C)$, are just the nodes in an equivalence class with respect to this relation and whose edges are those edges $(p, q)$ of $G$ with $p, q \in N(C)$.

We say $G$ is strongly connected if each pair of nodes of $G$ are strongly connected.

If $\mathcal{P}_{1}\left(p_{1}, p_{2}\right)$ and $\mathcal{P}_{2}\left(p_{2}, p_{3}\right)$ are paths of $G$, we define $\mathcal{P}_{1}+\mathcal{P}_{2}$ to be the path $\rho_{3}\left(p_{1}, p_{3}\right)$ obtained by concatenating the sequences of nodes in $P_{1}$ and $P_{2}$. This sum is clearly associative and $n\left(\mathcal{P}_{3}\right)=n\left(\mathcal{P}_{1}\right)+n\left(\mathcal{P}_{2}\right)$.

We define a cycle $\mathcal{C}$ to be a path $\rho(p, p)$.

We define $d(G)=\operatorname{gcd}(n(\mathcal{e}))$, taken over all cycles of $G$.

We define the product $G \times H$ of two graphs $G, H$ as follows

(i) $N(G \times H)=\{(p, q) \mid p \in N(G)$ and $q \in N(H)\}$,

Received by the editors May 26, 1962. 
(ii) $E(G \times H)=\left\{\left(\left(p_{1}, q_{1}\right),\left(p_{2}, q_{2}\right)\right) \mid\left(p_{1}, p_{2}\right) \in E(G)\right.$ and $\left(q_{1}, q_{2}\right)$ $\in E(H)\}$.

It may be readily seen that the adjacency matrix of $G \times H$ is just the Kronecker product of the adjacency matrices of $G$ and $H$. Since the product is clearly associative we may define unambiguously $G_{1} \times G_{2} \times \cdots \times G_{r}$.

\section{Preliminary lemmas.}

Lemma 1. Let $\mathcal{P}_{1}(p, q), \mathcal{P}_{2}(p, q)$ be paths of a strongly connected graph $G$. Then

$$
d(G) \mid\left(n\left(\mathcal{P}_{1}\right)-n\left(\mathcal{P}_{2}\right)\right) .
$$

Proof. Since $G$ is strongly connected there is a path $\odot_{3}(q, p)$. Then $\mathcal{P}_{1}+\mathcal{P}_{3}$ and $\mathcal{P}_{2}+\mathcal{P}_{3}$ are cycles; hence $d(G) \mid n\left(\mathcal{P}_{i}+\mathcal{P}_{3}\right)=n\left(\mathcal{P}_{i}\right)+n\left(\mathcal{P}_{3}\right)$, $(i=1,2)$. The result of the lemma now follows.

Let $G$ be a strongly connected graph and let $Z_{d}$ be the ring of residue classes mod $d=d(G)$. We may define a function $f: N(G) \rightarrow Z_{d}$ as follows: choose some node $p_{0}$ of $G$. Then for any $p \in N(G)$ define $f(p)=n\left(\odot\left(p_{0}, p\right)\right)$ for any path $\odot\left(p_{0}, p\right)$. Since $G$ is strongly connected there is some such path and Lemma 1 ensures that $f(p)$ is single valued.

Lemma 2. Let $G$ be a strongly connected graph and $f$ be defined as above. Then for any path $\mathcal{P}(p, q)$,

$$
n(\mathcal{P}) \equiv f(p)-f(q)(\bmod d(G)) .
$$

Proof. Let $\mathcal{P}_{1}\left(p_{0}, p\right)$ be a path and let $\boldsymbol{P}_{2}\left(p_{0}, q\right)=\boldsymbol{P}_{1}\left(p_{0}, p\right)+\propto(p, q)$. By definition of $f$,

$$
n\left(\odot_{1}\right) \equiv f(p)(\bmod d(G))
$$

and

$$
n\left(P_{2}\right) \equiv f(q)(\bmod d(G)) .
$$

The result of the lemma now follows since $n\left(\mathcal{P}_{2}\right)=n(\mathcal{P})+n\left(\mathcal{Q}_{1}\right)$.

Lemma 3. Let $G$ be a strongly connected graph and let $d=d(G)$. Then there exists a finite set of cycles $\mathfrak{C}_{1}, \cdots, \mathfrak{e}_{t}$ such that

$$
\underset{1 \leqq i \leqq \imath}{\operatorname{gcd}}\left(n\left(\mathfrak{e}_{i}\right)\right)=d .
$$

Proof. We may construct the set $\mathfrak{C}_{1} \cdots \mathfrak{e}_{t}$ inductively. Suppose $\mathfrak{C}_{1}, \cdots, \mathfrak{C}_{r}$ already chosen with $\operatorname{gcd}_{1 \leqq i \leqq r}\left(n\left(\mathfrak{C}_{i}\right)\right)=d_{r}>d$. Then there exists some $\mathfrak{e}$ with $d_{r} \nmid n(\mathfrak{e})$. Let this be $\mathfrak{C}_{r+1}$; we now have $d_{r}>d_{r+1}$. 
Since $\left\{d_{r}\right\}$ is a strictly monotonically decreasing sequence it must reach $d$ in a finite number of steps.

Lemma 4. Let $G$ be a strongly connected graph; let $d=d(G)$. Let $p_{1}, p_{2} \in N(G)$. Then there is an integer $n_{0}$ such that for all $n>n_{0}$ there is a path $\odot\left(p_{1}, p_{2}\right)$ with

$$
n(\odot)=n d+f\left(p_{2}\right)-f\left(p_{1}\right) .
$$

Proof. Let $\mathfrak{C}_{1}, \mathfrak{C}_{2}, \cdots, \mathfrak{C}_{t}$ be a set of cycles with $\operatorname{gcd}_{1 \leqq i \leqq t}\left(n\left(\mathfrak{C}_{i}\right)\right)$ $=d$. The existence of such a set is assured by Lemma 3. Let $n_{i}=n\left(\mathfrak{C}_{i}\right)$. By definition of the gcd, there exist integers $u_{1}, u_{2}, \cdots, u_{t}$ such that

$$
\sum_{i=1}^{t} u_{i} n_{i}=d
$$

Let $\mathfrak{C}_{i}=\mathcal{P}_{i}\left(q_{i}, q_{i}\right)$. Choose some set of paths $\mathcal{P}_{0}^{\prime}\left(p_{1}, q_{1}\right), \boldsymbol{P}_{1}^{\prime}\left(q_{1}, q_{2}\right), \cdots$, $\mathcal{P}_{t}^{\prime}\left(q_{t}, p_{2}\right)$. Let $n\left(\sum_{i=0}^{t} \mathcal{P}_{i}^{\prime}\right)=\sum_{i=0}^{t} n\left(\mathcal{P}_{i}^{\prime}\right)=K$. Then by Lemma 2 ,

$$
K=a d+f\left(p_{2}\right)-f\left(p_{1}\right)
$$

for some integer $a$.

Let $u=\max _{i}\left(\left|u_{i}\right|\right)$. Let $s=\sum_{i=1}^{n} n_{i} / d ; s$ is integral since $d \mid n_{i}$ for every $i$. We will show that $n_{0}=a+s+s^{2} u$ satisfies the requirements of the lemma. Let $n>n_{0}$. Then for some integral $b, c$

$$
n-a=b s+c
$$

with

$$
0 \leqq c<s
$$

Let

$$
v_{i}=b+c u_{i}
$$

Then

$$
\begin{aligned}
v_{i} & \geqq\left(\frac{n-a}{s}-1\right)-u s, & & \text { from (4) and (5) } \\
& \geqq \frac{n_{0}-a}{s}-1-u s, & & \text { since } n>n_{0} \\
& =0 & & \text { by definition of } n_{0} .
\end{aligned}
$$

I.e., the $v_{i}$ are non-negative integers. Hence we may construct a path

$$
\begin{aligned}
\rho\left(p_{1}, p_{2}\right)= & \rho_{0}^{\prime}\left(p_{1}, q_{1}\right)+v_{1} \rho_{1}\left(q_{1}, q_{1}\right)+\rho_{1}^{\prime}\left(q_{1}, q_{2}\right)+\cdots \\
& +v_{t} \odot_{t}\left(q_{t}, q_{t}\right)+\rho_{t}^{\prime}\left(q_{t}, p_{2}\right) .
\end{aligned}
$$

Then 


$$
\begin{aligned}
n(\mathcal{P}) & =\sum_{i=0}^{t} n\left(\mathcal{\odot}_{i}^{\prime}\right)+\sum_{i=1}^{t} v_{i} n\left(\mathcal{\odot}_{i}\right) \\
& =K+\sum_{i=1}^{t} v_{i} n_{i} \\
& =a d+f\left(p_{2}\right)-f\left(p_{1}\right)+b \sum_{i=1}^{t} n_{i}+\sum_{i=1}^{t} c u_{i} n_{i}, \text { from (3) and (6) } \\
& =a d+f\left(p_{2}\right)-f\left(p_{1}\right)+b s d+c d, \text { by }(2) \text { and the definition of } s \\
& =n d+f\left(p_{2}\right)-f\left(p_{1}\right) .
\end{aligned}
$$

This completes the proof of the lemma.

\section{Main theorems.}

THEOREM 1. Let $G$ and $H$ be strongly connected graphs. Let $d_{1}=d(G)$, $d_{2}=d(H), d_{3}=\operatorname{gcd}\left(d_{1}, d_{2}\right)$ and $D=\operatorname{lcm}\left(d_{1}, d_{2}\right)$. Then, in $G \times H$,

(i) $(p, q) \rightarrow\left(p^{\prime}, q^{\prime}\right)$ if and only if

$$
f_{G}(p)-f_{H}(q) \equiv f_{G}\left(p^{\prime}\right)-f_{H}\left(q^{\prime}\right)\left(\bmod d_{3}\right) .
$$

(ii) The number of components of $G \times H$ is $d_{3}$.

(iii) If $C$ is any component of $G \times H$ then $d(C)=D$.

Proof. (i) If $(p, q) \rightarrow\left(p^{\prime}, q^{\prime}\right)$ then there exists a path $\left\{\left(p_{i}, q_{i}\right)\right\}$ with $\left(p_{0}, q_{0}\right)=(p, q)$ and $\left(p_{n}, q_{n}\right)=\left(p^{\prime}, q^{\prime}\right)$. Then $\left\{p_{i}\right\}$ and $\left\{q_{i}\right\}$ are paths $\odot, \rho^{\prime}$ of $G$ and $H$ respectively with $n(\odot)=n\left(\odot^{\prime}\right)=n$. Hence, by Lemma 1,

$$
n \equiv f_{G}\left(p^{\prime}\right)-f_{G}(p)\left(\bmod d_{1}\right)
$$

and

$$
n \equiv f_{H}\left(q^{\prime}\right)-f_{H}(q)\left(\bmod d_{2}\right) .
$$

Therefore, since $d_{3} \mid d_{1}, d_{2}$,

$$
f_{G}\left(p^{\prime}\right)-f_{G}(p) \equiv f_{H}\left(q^{\prime}\right)-f_{H}(q)\left(\bmod d_{3}\right)
$$

Conversely, if (7) is satisfied, let $n_{1}$ be a value of $n_{0}$ satisfying the conclusions of Lemma 4 for $G, p, p^{\prime}$; let $n_{2}$ be defined similarly for $H, q, q^{\prime}$. Let $a, b$ be integers such that $a d_{1}-b d_{2}=d_{3}$. Let

$$
\frac{f_{G}(p)-f_{H}(q)-f_{G}\left(p^{\prime}\right)+f_{H}\left(q^{\prime}\right)}{d_{3}}=u,
$$

which is integral by hypothesis. Choose $r$ so that

$$
\left(a u+r d_{2}\right) \geqq n_{1}
$$


and

$$
\left(b u+r d_{1}\right) \geqq n_{2} .
$$

Then by Lemma 4 there is a path $\odot_{1}\left(p, p^{\prime}\right)$ of $G$ of length

$$
n\left(\odot_{1}\right)=\left(a u+r d_{2}\right) d_{1}+f_{G}\left(p^{\prime}\right)-f_{G}(p)
$$

and a path $\odot_{2}\left(q, q^{\prime}\right)$ of $H$ of length

$$
n\left(\odot_{2}\right)=\left(b u+r d_{1}\right) d_{2}+f_{H}\left(q^{\prime}\right)-f_{H}(q) .
$$

Then

$$
\begin{aligned}
n\left(\odot_{2}\right)-n\left(\odot_{1}\right) & =\left(a d_{1}-b d_{2}\right) u-u d_{3}, \quad \text { by }(8) \\
& =0 .
\end{aligned}
$$

Hence $\mathcal{P}_{1} \equiv\left\{p_{i}\right\}$ and $\mathcal{P}_{2} \equiv\left\{q_{i}\right\}$ are paths of the same length and so $\left\{\left(p_{i}, q_{i}\right)\right\}$ is a path of $G \times H$ whose end-nodes are just $(p, q)$ and $\left(p^{\prime}, q^{\prime}\right)$, i.e., $(p, q) \rightarrow\left(p^{\prime}, q^{\prime}\right)$ in $G \times H$.

(ii) Let $l(p, q)=f_{G}(p)-f_{H}(q)$, reduced $\bmod d_{3}$. Then, by (i), nodes $(p, q)$ and $\left(p^{\prime}, q^{\prime}\right)$ are strongly connected if and only if $l(p, q)=l\left(p^{\prime}, q^{\prime}\right)$. For any residue $x\left(\bmod d_{3}\right)$ there is certainly some node $(p, q)$ with $l(p, q)=x$. Hence the number of components of $G \times H$ is just $d_{3}$, each component consisting of just these nodes with the same value of $l(p, q)$.

(iii) Let $(p, q),\left(p^{\prime}, q^{\prime}\right)$ be nodes of $G \times H$ in the same component, $C$. Let $d_{0}=d(C)$. By Lemmas 1 and 4 the lengths of paths $\rho\left(p, p^{\prime}\right)$ in $G$ consist of all but a finite number of the integers

$$
n d_{1}+f_{G}\left(p^{\prime}\right)-f_{G}(p) \quad \text { for } n>0 .
$$

Similarly the lengths of paths $\odot\left(q, q^{\prime}\right)$ in $H$ consist of all but a finite number of the integers

$$
n d_{2}+f_{H}\left(q^{\prime}\right)-f_{H}(q) \quad \text { for } n>0 .
$$

The integers common to the arithmetic series (9) and (10) form an arithmetic series of difference $D=\operatorname{lcm}\left(d_{1}, d_{2}\right)$. Hence the lengths of paths $P\left((p, q),\left(p^{\prime}, q^{\prime}\right)\right)$ in $C$ consist of all but a finite number of the integers of this series; but applying Lemmas 1 and 4 to $G \times H$ the lengths of these paths consist of all but a finite number of the integers

$$
n d(C)+f_{C}\left(p^{\prime}, q^{\prime}\right)-f_{C}(p, q), \quad(n>0) .
$$

Therefore $d(C)=D=\operatorname{lcm}\left(d_{1}, d_{2}\right)$.

TheOREM 2. Let $G_{1}, G_{2}, \cdots, G_{s}$ be strongly connected graphs. Let $d_{i}=d\left(G_{i}\right)(i=1,2, \cdots, s)$. Then in $G_{1} \times G_{2} \times \cdots \times G_{s}$ : 
(i) The number of components is $\prod d_{i} / \mathrm{lcm}\left(d_{i}\right)$.

(ii) Each component $C$ has $d(C)=\operatorname{lcm}\left(d_{i}\right)$.

Proof. The results are true trivially for $s=1$. We shall complete the proof by induction. Suppose the results are true for $s=r$; i.e., $H \equiv G_{1} \times G_{2} \times \cdots \times G_{r}$ has $m$ components, each component $C$ having $d(C)=d_{H}$, where

$$
d_{H}=\underset{1 \leqq i \leqq r}{\operatorname{lcm}}\left(d_{i}\right)
$$

and

$$
m=\frac{\prod_{i=1}^{r} d_{i}}{d_{H}}
$$

In $H \times G_{r+1}$ nodes $(p, q)$ and $\left(p^{\prime}, q^{\prime}\right)$ are strongly connected only if $p$ and $p^{\prime}$ are strongly connected in $H$. Hence the components of $H \times G_{r+1}$ consist of just the components of $C \times G_{r+1}$ for all components $C$ of $H$. To each such product $C \times G_{r+1}$ we may apply the results of Theorem 1 . Each component, $C^{\prime}$, of $C \times G_{r+1}$ has

$$
\begin{aligned}
d\left(C^{\prime}\right) & =\operatorname{lcm}\left(d_{H}, d_{r+1}\right) \\
& =\operatorname{lcm}\left(\operatorname{lcm}\left(d_{1}, d_{2}, \cdots, d_{r}\right), d_{r+1}\right) \\
& =\operatorname{lcm}\left(d_{1}, d_{2}, \cdots, d_{r+1}\right) .
\end{aligned}
$$

The number of components of $C \times G$ is

$$
\operatorname{gcd}\left(d_{H}, d_{r+1}\right)=\frac{d_{H} \cdot d_{r+1}}{\operatorname{lcm}\left(d_{H}, d_{r+1}\right)} .
$$

Hence the number of components of $H \times G_{r+1}$

$$
\begin{aligned}
& =m \frac{d_{H} \cdot d_{r+1}}{\operatorname{lcm}\left(d_{H}, d_{r+1}\right)} \\
& =\frac{\prod_{i=1}^{r} d_{i}}{d_{H}} \cdot \frac{d_{H} \cdot d_{r+1}}{\operatorname{lcm}\left(d_{H}, d_{r+1}\right)} \\
& =\frac{\prod_{i=1}^{r+1} d_{i}}{\operatorname{lcm}\left(d_{1}, d_{2}, \cdots, d_{r+1}\right)} .
\end{aligned}
$$

Hence the results of the theorem are true for $s=r+1$. The theorem 
now follows by induction.

We may apply the results of Theorems 1 and 2 to undirected graphs by replacing each edge $(p, q)$ of the undirected graph by a pair of edges $(p, q),(q, p)$ and constructing an equivalent directed graph. For such a graph $G, d(G)$ is 1 or 2 according as $G$ has or has not an odd cycle. Theorem 1 of [1] now follows from Theorem 1 above. From Theorem 2 follows directly

Theorem 3. Let $G_{1}, G_{2}, \cdots, G_{r}$ be undirected graphs. If exactly s of these graphs have an odd cycle then $m$, the number of components of $G_{1} \times G_{2} \times \cdots \times G_{r}$ is given by

$$
m= \begin{cases}1 & s \geqq r-1 \\ 2^{r-s} & s \leqq r-1 .\end{cases}
$$

BiBLIOGRAPHY

1. Claude Berge, Thêorie des graphes et ses applications, Dunod, Paris, 1958.

2. P. M. Weichsel, The Kronecker product of graphs, Proc. Amer. Math. Soc. 13 (1962), 47-52.

International Business Machines Corporation 